\title{
Extended TODIM Method for MADM Problem under Trapezoidal Intuitionistic Fuzzy Environment
}

\author{
H.P. Ren, M.F. Liu, H. Zhou
}

\author{
Haiping Ren* \\ Teaching Department of Basic Subjects \\ Jiangxi University of Science and Technology,330013 Nanchang, China \\ *Corresponding author: 9520060004@jxust.edu.cn

\section{Manfeng Liu} \\ The Collaborative Innovation Center \\ Jiangxi University of Finance and Economics,330013 Nanchang, China \\ liumanfeng@sina.com
}

\section{Hui Zhou}

School of Mathematics and Computer Science

Yichun University,336000 Yichun, China

huihui7978@126.com

\begin{abstract}
In actual decision making process, the final decision result is often affected by decision maker's psychological behavior, however, for the multiple attribute decision making (MADM) problem in which attributes values are expressed with trapezoidal intuitionistic fuzzy numbers, there are few literatures considering the decision maker's behavior factors in decision making process. For this case, this paper first proposes a new distance measure of TIFNs and a new ranking method which considers decision maker's attitude behavior, and then develops an extended TODIM decision making method. Finally an example is given to illustrate the validity and practicability of the proposed method.

Keywords: TODIM method, trapezoidal intuitionistic fuzzy number, multiple attribute decision making, ranking method.
\end{abstract}

\section{Introduction}

In recent years, with the increasing complexity of the managerial decision making environment, many managerial decision-making problems contain qualitative properties which are difficult to quantify. Zadeh's fuzzy sets have been greatly successful in dealing with fuzzy management decision making problems $[3,4,18,20,22,35]$. Zadeh's fuzzy set is characterized by a single scale (membership), which can only characterize the support and opposition of the two aspects of the evidence. But some decision making problems have ambiguous hesitant phenomenon with respect to evaluation of information, and Zadeh's fuzzy set is hard or difficult to depict these situations. Therefore, many scholars developed Zadeh's fuzzy set, and intuitionistic fuzzy (IF) set is one of the most famous fuzzy sets among them. Originally proposed by Atanassov in 1986 [1], IF sets can well describe the hesitation and uncertainty of judgment through the addition of a non-membership parameters, which can describe the vague characters of things comprehensively. Then IF sets have become a powerful and effective tool in dealing with uncertain or vague information in actual applications. In dealing with ambiguity and uncertainty, IF sets are more flexible and practical than fuzzy sets, and thus they have been applied widely in decision making.

Because of the complexity and uncertainty of objective things and the limitation of decision maker's knowledge, membership and non-membership functions are sometimes difficult to represent by using the precise numbers. But interval number can be very useful to describe this kind 
of case, so Atanassov and Gargov [2] extended IF sets to interval-valued IF sets. Some scholars put forward the concept of continuous IF numbers to describe an uncertain quantity or a difficult quantification number on the basis of the concept of IF set. Grzegrorzewski [10] extended IF sets to the continuous case of IF numbers . Nehi and Maleki [21] put forward trapezoidal intuitionistic fuzzy number (TIFN), and defined the corresponding operation rules, which caused great concern in the academic community. Shu [27] proposed the definition of triangular intuitionistic fuzzy number, which is a special example of TIFN, and they put it to the application of fault tree analysis, base on these research, Wang and Zhang [31] further expanded it and gave the definition of a generalized TIFN. Different from the definitions of IF sets, TIFN is added to a trapezoidal fuzzy number, which makes the membership degree and the non-membership degree no longer be only a fuzzy concept Good or Excellent; then, the assessment information given by the decision makers can be expressed exactly. Comparing with IF sets, they have more attractive explanation, and are easy to be quantified and executed by the decision maker, and thus they have more theoretical value in the field of decision science $[8,14,16,29]$.

At present, the theory and application of fuzzy numbers, such as triangular intuitionistic fuzzy number, trapezoidal intuitionistic fuzzy number, have been received great attention. But most of the existing decision-making methods do not consider the influence of the behavior of the decision-makers in the decision process, because they are assumed that the decision maker is completely rational. However, the actual decision-making process is often accompanied by the different psychological behavior of the decision-makers and the attitude of the risk and other factors of behavior. Kahneman and Tversky [13] proposed the prospect theory, which can describe the decision maker's psychological behavior. Based on prospect theory, Gomes and Lima ( $[6,7]$ ) developed a new MADM method named TODIM method, which has made many successful applications, such as material evaluation [34], green supplier selection [26], logistics outsourcing [30] etc. Fan et al. [5] proposed an extension of TODIM (H-TODIM) to solve the hybrid MADM problems in which attribute values have three forms: crisp numbers, interval numbers and fuzzy numbers. Qin [23] proposed a generalization of the TODIM method under triangular intuitionistic fuzzy environment. Ren et al. [25] extended the TODIM method to deal with the MADM problem in which attribute values are expressed with Pythagorean fuzzy numbers. Zhang et al. [33] developed the TODIM method to solve the MADM problem in which the attribute values are expressed with neutrosophic numbers. In this paper, we will develop a new extension of TODIM method to solve the MADM problem in which attribute values are expressed with TIFNs, and an application example is used to illustrate the validity and practicability of the proposed method.

\section{Preliminary knowledge}

\subsection{Definitions of TIFNs}

Firstly, we recall the definition of the TIFN and the related theory. In order to use the concept of IF sets to define an uncertain number or difficult to quantify the amount, Grzegrorzewski [10] extended the IF sets to the continuous case of the IF sets, and gave the following definition:

Definition 8. Let $R$ be the set of real numbers, $A$ is called an IF number in $R$, if its membership function $\mu_{A}(x)$ and non-membership function $\nu_{A}(x)$ are respectively defined as follows ( [10]) : 


$$
\mu_{A}(x)=\left\{\begin{array}{c}
0, \quad x<a_{1} \\
f_{A}(x), \quad a_{1} \leq x \leq a_{2} \\
1, \quad a_{2} \leq x \leq a_{3} \\
g_{A}(x), \quad a_{3} \leq x \leq a_{4} \\
0, \quad a_{4}<x
\end{array}\right.
$$

and

$$
\nu_{A}(x)=\left\{\begin{array}{c}
1, \quad x<b_{1} \\
h_{A}(x), \quad b_{1} \leq x \leq b_{2} \\
0, \quad b_{2} \leq x \leq b_{3} \\
k_{A}(x), \quad b_{3} \leq x \leq b_{4} \\
1, \quad a_{b}<x
\end{array}\right.
$$

where $0 \leq \mu_{A}(x)+\nu_{A}(x) \leq b_{2}, a_{i}, b_{i} \in R, i=1,2,3,4$, and they satisfy $b_{1} \leq a_{1} \leq$ $b_{2} \leq a_{2} \leq b_{3} \leq a_{3} \leq b_{4} \leq a_{4}$. The four functions $f_{A}(x), g_{A}(x), h_{A}(x)$ and $k_{A}(x)$ are real value aunctions defined in interval $[0,1]$. The functions $f_{A}(x), k_{A}(x)$ are non-decreasing coctinuous functions and $g_{A}(x), h_{A}(x)$ are non-increasing continuous functions.

On the basis of Grzegrorzewski's IF numbers, Nehi [21] developed the TIFN in 2005, which is given in Definition 2.

Definition 9. Let $b_{1} \leq a_{1} \leq b_{2} \leq a_{2} \leq b_{3} \leq a_{3} \leq b_{4} \leq a_{4} \in R$. A fuzzy number $A$ is called a TIFN, if its membership function $\mu_{A}(x)$ and non-membership function $\nu_{A}(x)$ are respectively defined as follows( [21]):

$$
\mu_{A}(x)=\left\{\begin{array}{c}
0, \quad x<a_{1} \\
\frac{x-a_{1}}{a_{2}-a_{1}}, \quad a_{1} \leq x \leq a_{2} \\
1, \quad a_{2} \leq x \leq a_{3} \\
\frac{a_{4}-x}{a_{4}-a_{3}}, \quad a_{3} \leq x \leq a_{4} \\
0, \quad a_{4}<x
\end{array}\right.
$$

and

$$
\nu_{A}(x)=\left\{\begin{array}{c}
1, \quad x<b_{1} \\
\frac{x-b_{1}}{b_{2}-b_{1}}, \quad b_{1} \leq x \leq b_{2} \\
0, \quad b_{2} \leq x \leq b_{3} \\
\frac{b_{4}-x}{b_{4}-b_{3}}, \quad b_{3} \leq x \leq b_{4} \\
1, \quad a_{b}<x
\end{array}\right.
$$

We denote $A$ by $A=<\left(a_{1}, a_{2}, a_{3}, a_{4}\right),\left(b_{1}, b_{2}, b_{3}, b_{4}\right)>$.

Definition 10. For two TIFNs $A_{1}=<\left(a_{11}, a_{12}, a_{13}, a_{14}\right),\left(b_{11}, b_{12}, b_{13}, b_{14}\right)>$ and $A_{2}=<$ $\left(a_{21}, a_{22}, a_{23}, a_{24}\right),\left(b_{21}, b_{22}, b_{23}, b_{24}\right)>$, the operational laws are defined as follows [2]:

(1) $A_{1}+A_{2}=<\left(a_{11}+a_{21}, a_{12}+a_{22}, a_{13}+a_{23}, a_{14}+a_{24}\right),\left(b_{11}+b_{21}, b_{12}+b_{22}, b_{13}+b_{23}, b_{14}+b_{24}\right)>$

(2) $k A_{1}=<\left(k a_{11}, k a_{12}, k a_{13}, k a_{14}\right),\left(k b_{11}, k b_{12}, k b_{13}, k b_{14}\right)>$, for $k>0$

Usually, $\alpha$-cut set is a very effective tool for describing the number of fuzzy numbers [11], and TIFNs have two classes $\alpha$-cut sets: $\left(A^{+}\right)_{\alpha}$ and $\left(A^{-}\right)_{\alpha}$.

Definition 11. Let $A=<\left(a_{1}, a_{2}, a_{3}, a_{4}\right),\left(b_{1}, b_{2}, b_{3}, b_{4}\right)>$ be a TIFN, then the two classes $\alpha$-cut sets $\left(A^{+}\right)_{\alpha}$ and $\left(A^{-}\right)_{\alpha}$ are defined as follows, respectively:

$$
\begin{gathered}
\left(A^{+}\right)_{\alpha}=\left\{x \in R \mid \mu_{A}(x) \geq \alpha\right\} \\
\left(A^{-}\right)_{\alpha}=\left\{x \in R \mid 1-\nu_{A}(x) \geq \alpha\right\}
\end{gathered}
$$


According to Definition 4 , each $\alpha$-cut set is a closed interval,thus they can be denoted as $\left(A^{+}\right)_{\alpha}=\left[\left(A_{L}^{+}\right)_{\alpha},\left(A_{U}^{+}\right)_{\alpha}\right]$ and $\left(A^{-}\right)_{\alpha}=\left[\left(A_{L}^{-}\right)_{\alpha},\left(A_{U}^{-}\right)_{\alpha}\right]$, where

$$
\begin{gathered}
\left(A_{L}^{+}\right)_{\alpha}=\inf \left\{x \in R \mid \mu_{A}(x) \geq \alpha\right\}, \\
\left(A_{U}^{+}\right)_{\alpha}=\sup \left\{x \in R \mid \mu_{A}(x) \geq \alpha\right\}, \\
\left(A_{L}^{-}\right)_{\alpha}=\inf \left\{x \in R \mid 1-\nu_{A}(x) \geq \alpha\right\}, \\
\left(A_{U}^{-}\right)_{\alpha}=\sup \left\{x \in R \mid 1-\nu_{A}(x) \geq \alpha\right\} .
\end{gathered}
$$

Then, for a TIFN $A=<\left(a_{1}, a_{2}, a_{3}, a_{4}\right),\left(b_{1}, b_{2}, b_{3}, b_{4}\right)>$, we can easily derive the following results:

$$
\begin{gathered}
\left(A^{+}\right)_{\alpha}=\left[\left(A_{L}^{+}\right)_{\alpha},\left(A_{U}^{+}\right)_{\alpha}\right]=\left[a_{1}+\left(a_{2}-a_{1}\right) \alpha, a_{4}-\left(a_{4}-a_{3}\right) \alpha\right], \\
\left(A^{-}\right)_{\alpha}=\left[\left(A_{L}^{-}\right)_{\alpha},\left(A_{U}^{-}\right)_{\alpha}\right]=\left[b_{1}+\left(b_{2}-b_{1}\right)(1-\alpha), b_{4}-\left(b_{4}-b_{3}\right)(1-\alpha)\right],
\end{gathered}
$$

\subsection{A novel distance measure of TIFNs}

In the follow we will develop a novel distance of TIFNs based on the following distance measure between two fuzzy numbers proposed by Grzegorzewski in 1998.

Lemma 2.1. For two any fuzzy numbers $A$ and $B$, and the corresponding $\alpha$-cut sets are respectively $\left[\left(A_{L}^{+}\right)_{\alpha},\left(A_{U}^{+}\right)_{\alpha}\right]$ and $\left[\left(A_{L}^{-}\right)_{\alpha},\left(A_{U}^{-}\right)_{\alpha}\right]$, then the distance measure between them is defined as [9].

$$
d(A, B)=\left(\int_{0}^{1}\left(\left(A_{L}\right)_{\alpha}-\left(B_{L}\right)_{\alpha}\right)^{2} d \alpha+\int_{0}^{1}\left(\left(A_{U}\right)_{\alpha}-\left(B_{U}\right)_{\alpha}\right)^{2} d \alpha\right)^{1 / 2} .
$$

Inspired by the Lemma 1, we define the following distance measure for two arbitrary TIFNs and as follows:

$$
\begin{aligned}
d(A, B)=\frac{1}{2}\left(\int_{0}^{1}\left(\left(A_{L}^{+}\right)_{\alpha}-\left(B_{L}^{+}\right)_{\alpha}\right)^{2}+\left(\left(A_{U}^{+}\right)_{\alpha}-\left(B_{U}^{+}\right)_{\alpha}\right)^{2} d \alpha\right)^{1 / 2} & \\
+ & \frac{1}{2}\left(\int_{0}^{1}\left(\left(A_{L}^{-}\right)_{\alpha}-\left(B_{L}^{-}\right)_{\alpha}\right)^{2}+\left(\left(A_{U}^{-}\right)_{\alpha}-\left(B_{U}^{-}\right)_{\alpha}\right)^{2} d \alpha\right)^{1 / 2}
\end{aligned}
$$

It is easy to prove that the new distance measure can satisfy the non negativity, symmetry and triangle inequality. By straightforward calculation, we can get Theorem 1.

Theorem 1. Let $A_{1}=<\left(a_{11}, a_{12}, a_{13}, a_{14}\right),\left(b_{11}, b_{12}, b_{13}, b_{14}\right)>$ and $A_{2}=<\left(a_{21}, a_{22}, a_{23}, a_{24}\right)$, $\left(b_{21}, b_{22}, b_{23}, b_{24}\right)>$ be two TIFNs, then the distance measure between $A_{1}$ and $A_{2}$ is defined as follows:

$$
d\left(A_{1}, A_{2}\right)=\frac{1}{2}\left[\left(I_{1}+I_{2}\right)^{1 / 2}+\left(I_{3}+I_{4}\right)^{1 / 2}\right]
$$

Proof: Here

$$
\begin{aligned}
I_{1} & =\int_{0}^{1}\left(\left(A_{1 L}^{+}\right)_{\alpha}-\left(A_{2 L}^{+}\right)_{\alpha}\right)^{2} d \alpha \\
& =\int_{0}^{1}\left[\left(a_{21}-a_{11}\right)+\left(a_{22}-a_{21}-a_{12}+a_{11}\right) \alpha\right]^{2} d \alpha \\
& =\int_{0}^{1}[x+(y-x) \alpha]^{2} d \alpha \\
& =x^{2}+x(y-x)+\frac{1}{3}(y-x)^{2} \\
& =\frac{1}{3}\left(x^{2}+x y+y^{2}\right) \\
& =\frac{1}{3}\left(\left(a_{21}-a_{11}\right)^{2}+\left(a_{21}-a_{11}\right)\left(a_{22}-a_{12}\right)+\left(a_{22}-a_{12}\right)^{2}\right)
\end{aligned}
$$

where $x=a_{21}-a_{11}, y=a_{22}-a_{12}$. Similarly, we have

$$
\begin{aligned}
I_{2} & =\int_{0}^{1}\left(\left(A_{1 U}^{+}\right)_{\alpha}-\left(A_{2 U}^{+}\right)_{\alpha}\right)^{2} d \alpha \\
& =\frac{1}{3}\left(\left(a_{23}-a_{13}\right)^{2}+\left(a_{23}-a_{13}\right)\left(a_{24}-a_{14}\right)+\left(a_{24}-a_{14}\right)^{2}\right)
\end{aligned}
$$




$$
\begin{aligned}
I_{3} & =\int_{0}^{1}\left(\left(A_{1 L}^{-}\right)_{\alpha}-\left(A_{2 L}^{-}\right)_{\alpha}\right)^{2} d \alpha \\
& =\frac{1}{3}\left(\left(b_{21}-b_{11}\right)^{2}+\left(b_{21}-b_{11}\right)\left(b_{22}-b_{12}\right)+\left(b_{22}-b_{12}\right)^{2}\right) \\
I_{4} & =\int_{0}^{1}\left(\left(A_{1 U}^{-}\right)_{\alpha}-\left(A_{2 U}^{-}\right)_{\alpha}\right)^{2} d \alpha \\
& =\frac{1}{3}\left(\left(b_{23}-b_{13}\right)^{2}+\left(b_{23}-b_{13}\right)\left(b_{24}-b_{14}\right)+\left(b_{24}-b_{14}\right)^{2}\right)
\end{aligned}
$$

Then by lemma 1 , we can derive the conclusion (1).

\section{A new ranking function of TIFNs}

In actual decision making process, the final decision result is often affected by the different attitudes of decision-makers, although many scholars have already considered the influence of different attitude index for the MADM problems in which attributes values are expressed with interval number and triangular fuzzy number [17, 24,28]. However, for the MADM problem in which attributes values are expressed with TIFNs, and there is no literature considering the decision maker's attitude in decision making process. Thus, we take the decision maker's mentality into the decision-making process, and put forward a new TIFN ranking method.

Definition 12. Let $\tilde{a}=\left[a^{L}, a^{U}\right]$ be an interval fuzzy number, $M_{\tilde{a}}=0.5\left(a^{L}+a^{U}\right)$ and $D_{\tilde{a}}=$ $0.5\left(a^{U}-a^{L}\right) . F_{\tilde{a}}(\lambda):[0,1] \rightarrow \tilde{a}$ is a function of parameter with the following form:

$$
F_{\tilde{a}}(\lambda)=M_{\tilde{a}}+(2 \lambda-1) D_{\tilde{a}}=(1-\lambda) a^{L}+\lambda a^{U} .
$$

Here, the parameter $\lambda$ is called attitude index of interval number $\tilde{a}$.

Remark 3.1. Apparently, $F_{\tilde{a}}(\lambda)$ is a monotonic increasing function on interval[0,1]. When an attribute is a benefit type attribute, i.e. the value of it is the-larger-the-better. When $\lambda=$ 0 , then $F_{\tilde{a}}(0)=a^{L}=\left[a^{L}, a^{L}\right]$ is smaller than the fuzzy number $\tilde{a}=\left[a^{L}, a^{U}\right]$,thus for benefit type attribute, the parameter $\lambda=0$ demonstrates a pessimistic attitude. Similarly, $\lambda=1$ demonstrates an optimistic attitude and $\lambda=0.5$ demonstrates a moderate attitude.

Lemma 3.1. Let $\tilde{a}=\left(a^{L}, a^{M}, a^{U}\right)$ be a triangular fuzzy number, and for any real number $\alpha \in[0,1], \alpha-$ cut set of $\tilde{a}$ can be easily derived as follows [15]:

$$
\tilde{a}_{\alpha}=\left[a^{L}(\alpha), a^{U}(\alpha)\right]=\left[a^{L}+\left(a^{M}-a^{L}\right) \alpha, a^{U}-\left(a^{U}-a^{M}\right) \alpha\right]
$$

Remark 3.2. For two arbitrary triangular fuzzy number $\tilde{a}$ and $\tilde{b}, \alpha-$ cut sets are often used to compare them. Considering the $\alpha-c u t$ sets of triangular fuzzy numbers are still interval numbers, and $\alpha$ is an arbitrary value in interval $[0,1]$,to eliminate the arbitrariness and reflect the decision maker's attitude behavior, Ren and Liu [24]developed a new ranking function of triangular fuzzy number considering with attitude of decision maker(s) motivated by Definition 5 .

Definition 13. Let $\tilde{a}=\left(a^{L}, a^{M}, a^{U}\right)$ be a triangular fuzzy number, then for any parameter $\lambda \in[0,1]$, the function $F(\tilde{a}, \lambda)$ is a new ranking function of triangular fuzzy number considering attitude of decision maker(s) with the following formula [24]:

$$
F(\tilde{a}, \lambda)=\int_{0}^{1}(1-\lambda) a^{L}(\alpha)+\lambda a^{U}(\alpha) d \alpha
$$

Obviously, $F(\tilde{a}, \lambda)$ can be rewritten as the following form:

$$
\begin{gathered}
F(\tilde{a}, \lambda)=\int_{0}^{1}(1-\lambda)\left(a^{L}+\left(a^{M}-a^{L}\right) \alpha\right)+\lambda\left(a^{U}-\left(a^{U}-a^{M}\right) \alpha\right) d \alpha \\
=\left[(1-\lambda) a^{L}+a^{M}+\lambda a^{U}\right] / 2
\end{gathered}
$$


Let $r \in[0,1]$, then according to Definition 6, Ren and Liu [28] gave the following rule for comparing two triangular fuzzy numbers $\tilde{a}=\left(a^{L}, a^{M}, a^{U}\right)$ and $\tilde{b}=\left(b^{L}, b^{M}, b^{U}\right)$ :

(i) For arbitrary $\lambda \in[0,1]$, if $F(\tilde{a}, \lambda) \leq F(\tilde{b}, \lambda)$, then $\tilde{a}$ is smaller than $\tilde{b}$, and noted $\tilde{a} \leq \tilde{b}$;

(ii)For arbitrary $\lambda \in[0,1]$, if $F(\tilde{a}, \lambda)=F(\tilde{b}, \lambda)$, then $\tilde{a}$ is equal to $\tilde{b}$, and noted $\tilde{a}=\tilde{b}$;

(iii)For arbitrary $\lambda \in[0, r]$,if $F(\tilde{a}, \lambda) \leq F(\tilde{b}, \lambda)$,while when $\lambda \in[r, 1], F(\tilde{a}, \lambda) \geq F(\tilde{b}, \lambda)$; then for the decision maker whose attitude is pessimistic, the ranking result is $\tilde{a} \leq \tilde{b}$, which for the decision maker whose attitude is optimistic, the ranking result is $\tilde{a} \geq \tilde{b}$.

Motivated by Definition 6, we will develop a new ranking function of trapezoidal intuitionistic fuzzy number defined in Definition 7.

Definition 14. Let $A=<\left(a_{1}, a_{2}, a_{3}, a_{4}\right),\left(b_{1}, b_{2}, b_{3}, b_{4}\right)>$ be a TIFN, and $p(\alpha)$ is a real function defined on $[0,1]$, then a new ranking function $F(A, \lambda)$ including the attitude behavior of decision maker is defined as follows:

$$
\begin{array}{r}
F(A, \lambda)=\frac{1}{2} \int_{0}^{1}(1-\lambda)\left(A_{L}^{+}\right)_{\alpha}+\lambda\left(A_{U}^{+}\right)_{\alpha} d P(\alpha) \\
+\frac{1}{2} \int_{0}^{1}(1-\lambda)\left(A_{L}^{-}\right)_{\alpha}+\lambda\left(A_{U}^{-}\right)_{\alpha} d P(\alpha)
\end{array}
$$

Particularly, if $P(\alpha)=\alpha^{r+1}$, then we can get

$$
\begin{gathered}
F(A, \lambda)=\frac{1}{2(r+2)}\left[(1-\lambda)\left(a_{1}+b_{1}\right)+(r+1)(1-\lambda)\left(a_{2}+b_{2}\right)\right. \\
\left.+(r+1) \lambda\left(a_{3}+b_{3}\right)+\lambda\left(a_{4}+b_{4}\right)\right]
\end{gathered}
$$

Remark 3.3. If $r=0, \lambda=1 / 2$, then $F(A, \lambda)$ is as same as that ranking function of Ye [32]. Similar discussion with Remark 1, the parameter $\lambda$ is the attitude index. Then the ranking function $F(A, \lambda)$ can reflect the attitude behavior, and thus it can better depict the actual decision process with the help of different values of $\lambda$ than that ranking function of Ye [32].

Definition 15. For two given TIFNs $A_{1}$ and $A_{2}$, and $r \in[0,1]$, the relationship of $A_{1}$ and $A_{2}$ can be defined as follows:

(i) For any $\lambda \in[0,1]$, if $F\left(A_{1}, \lambda\right) \leq F\left(A_{2}, \lambda\right)$, then $A_{1}$ is smaller than $A_{2}$, and noted $A_{1} \leq A_{2}$;

(ii)For any $\lambda \in[0,1]$, if $F\left(A_{1}, \lambda\right)=F\left(A_{2}, \lambda\right)$,then $A_{1}$ is equal to $A_{2}$, and noted $A_{1}=A_{2}$;

(iii)For any $\lambda \in[0, r]$,if $F\left(A_{1}, \lambda\right) \leq F\left(A_{2}, \lambda\right)$,while when $\lambda \in[r, 1], F\left(A_{1}, \lambda\right) \geq F\left(A_{2}, \lambda\right)$; then for the decision maker whose attitude is pessimistic, the ranking result is $A_{1} \leq A_{2}$, while for the decision maker whose attitude is optimistic, the ranking result is $A_{1} \geq A_{2}$.

\section{Extended TODIM method for MADM under TIFN environ- ment}

For a given MADM problem, let $X=\left\{x_{1}, x_{2}, \cdots, x_{m}\right\}$ be a possible alternatives set, and $O=\left\{o_{1}, o_{2}, \cdots, o_{n}\right\}$ be the evaluation attribute set. $D=\left\{D_{1}, D_{2}, \cdots, D_{s}\right\}$ is the expert set. Suppose the rating of $x_{i}(i=1,2, \cdots, m)$ with respect to $o_{j}(j=1,2, \cdots, n)$ given by expert $D_{k}(k=1,2, \cdots, s)$ is a linguistic term noted by $\tilde{s}_{i j}^{k}$, which belongs to the linguistic terms set $\{$ Absolutely low, Low, Fairly low, Fairly high, High, Absolutely high $\}$. Then the MADM problem can be expressed with matrices $\tilde{S}^{k}=\left(s_{i j}^{k}\right)_{m \times n}, k=1,2, \ldots, s$.

Let $w=\left(w_{1}, w_{2}, \ldots, w_{n}\right)^{T}$ be the attribute weight vector, and each element $w_{j}$ represents the degree of importance of attribute, which can be given by decision maker or determined by some weighting methods, such as AHP method or entropy weighting method. 
The calculation steps of the extended TODIM method considering the decision maker's attitude are given as follows:

Step 1. According to Table 1 [32], $\tilde{s}_{i j}^{k}$ can be transformed with TIFNs $\tilde{a}_{i j}(k), i=$ $1,2, \ldots, m, j=1,2, \ldots, n$ and $k=1,2, \ldots, s$.

Table 1: Linguistic terms and corresponding TIFNs

\begin{tabular}{|c|c|}
\hline Linguistic terms & TIFNs \\
\hline Absolutely low $(\mathrm{AL})$ & $<(0.001,0.001,0.001,0.001),(0.001,0.001,0.001,0.001)>$ \\
\hline Low $(\mathrm{L})$ & $<(0.0,0.1,0.2,0.3),(0.0,0.1,0.2,0.3)>$ \\
\hline Fairly low $(\mathrm{FL})$ & $<(0.1,0.2,0.3,0.4),(0.0,0.2,0.3,0.5)>$ \\
\hline Medium $(\mathrm{M})$ & $<(0.3,0.4,0.5,0.6),(0.2,0.4,0.5,0.7)>$ \\
\hline Fairly high $(\mathrm{FH})$ & $<(0.5,0.6,0.7,0.8),(0.4,0.6,0.7,0.9)>$ \\
\hline High $(\mathrm{H})$ & $<(0.7,0.8,0.9,1.0),(0.7,0.8,0.9,1.0)>$ \\
\hline Absolutely high $(\mathrm{AH})$ & $<(1.0,1.0,1.0,1.0),(1.0,1.0,1.0,1.0)>$ \\
\hline
\end{tabular}

The linguistic terms decision matrices $\tilde{S}^{k}=\left(s_{i j}^{k}\right)_{m \times n}$ are transformed into trapezoidal intuitionistic fuzzy decision matrices $\tilde{A}^{k}=\left(\tilde{a}_{i j}^{k}\right)_{m \times n}(k=1,2, \ldots, s)$.

Step 2. Let $\tilde{s}_{i j}$ be the total score of alternative $x_{i}$ with respect to attribute $o_{j}$ given by all decision makers, and it is defined as

$$
\tilde{a}_{i j}=\frac{1}{s} \sum_{k=1}^{s} \tilde{a}_{i j}^{k} .
$$

Step 3. Determine the weights of evaluation attributes. Using the Definition 7 and $P(\alpha)=\alpha^{r+1}$, we can get the intuitionistic fuzzy sorting function matrix $F(\lambda)=$ $\left(F\left(\tilde{a}_{i j}, \lambda\right)\right)_{m \times n}$, where $F\left(\tilde{a}_{i j}, \lambda\right)$ is the ranking function of fuzzy number $\tilde{a}_{i j}$ considering with the attitude of decision maker. For the maximum TIFN $\tilde{a}^{*}=<(1,1,1,1),(1,1,1,1)>$, $F\left(\tilde{a}^{*}, \lambda\right)=1$.

Now, we will propose a new weighting method by means of the proposed ranking function. The reasonable weight should be the minimum of the total deviation of the alternative $x_{i}(i=1,2, \cdots, m)$ and the positive ideal solution $\tilde{a}^{*}$. Therefore, we can establish the following optimization model:

$$
\begin{aligned}
& \min G(w)=\sum_{j=1}^{n} \sum_{i=1}^{m} w_{j}\left(1-F\left(\tilde{a}_{i j}\right)\right) \\
& \text { s.t. }\left\{\begin{array}{l}
w \in H \\
\sum_{j=1}^{n} w_{j}=1 \\
w_{j} \geq 0, j=1,2, \ldots, n
\end{array}\right.
\end{aligned}
$$

By solving the Eq. (3), the optimal solution $w^{*}=\arg \max S$ is chosen as the optimal attribute weights.

Step 4. Calculate TODIM score as follows:

(i) Calculate $w_{r c}=\frac{w_{c}}{w_{r}}$, where the value $w_{r c}$ represents the weight value of criteria $r$ divided by the weight of the reference point $c$, and $w_{r}=\max _{1 \leq c \leq n}\left\{w_{c}\right\}$. 
(ii) For given value of attitude index $\lambda$, calculate

$$
\phi_{c}\left(x_{i}, x_{j}\right)=\left\{\begin{array}{c}
\sqrt{\frac{d\left(x_{i}, x_{j}\right)}{w_{r c}}}, \quad F\left(x_{i c}, \lambda\right)-F\left(x_{j c}, \lambda\right)>0 \\
0 \quad F\left(x_{i c}, \lambda\right)-F\left(x_{j c}, \lambda\right)=0 \\
-\frac{1}{\theta} \sqrt{\frac{d\left(x_{i}, x_{j}\right)}{w_{r c}}}, F\left(x_{i c}, \lambda\right)-F\left(x_{j c}, \lambda\right)<0
\end{array}\right.
$$

Here $d\left(x_{i}, x_{j}\right)=\sum_{c=1}^{n} w_{r c} d\left(\tilde{a}_{i c}, \tilde{a}_{j c}\right)$. Here the parameter $\theta$ is an important parameter in prospect theory, and $\theta>1$ shows that the individual is losses aversion, and $\theta<1$ shows the individuals are attenuated when facing the losses [19]. Here we set $\theta=2.25$, which is the most often used value of $\theta$ in prospect theory.

(iii) Let $\delta\left(A_{i}, A_{j}\right)=\sum_{c=1}^{n} \phi_{c}\left(A_{i}, A_{j}\right), i, j=1,2, \ldots, m$, calculate the comprehensive evaluation index value:

$$
\xi_{i}=\frac{\sum_{j=1}^{m} \delta\left(A_{i}, A_{j}\right)-\min _{1 \leq i \leq m} \sum_{j=1}^{m} \delta\left(A_{i}, A_{j}\right)}{\max _{1 \leq i \leq m} \sum_{j=1}^{m} \delta\left(A_{i}, A_{j}\right)-\min _{1 \leq i \leq m} \sum_{j=1}^{m} \delta\left(A_{i}, A_{j}\right)}, i=1,2, \cdots, m .
$$

Step 5. Rank the alternatives according to $\xi_{i}(i=1,2, \cdots, m)$ in decreasing order.

\section{Applied example}

Suppose that a company wants to invest a large amount of money in the best options(Herrera and Herrera-Viedma [12]; [32]). There are four parallel alternatives: $x_{1}(\mathrm{a}$ car company), $x_{2}$ (a food company), $x_{3}$ (a computer company), $x_{4}$ (an arms company) and three evaluation attributes $o_{1}$ (the risk analysis), $o_{2}$ (the growth analysis), and $o_{3}$ (the environmental impact analysis). The risk investment company now employs four experts to evaluate these four alternative enterprises. The evaluation values are expressed with linguistic terms, and the corresponding trapezoidal intuitionistic fuzzy evaluation decision matrices are listed in Table 2 to Table 4 . Our task is to choose the best investment plan by the method presented in this paper.

Table 2: Linguistic evaluation values given by expert 1

\begin{tabular}{|c|c|c|c|}
\hline Alternatives & $o_{1}$ & $o_{2}$ & $o_{3}$ \\
\hline$x_{1}$ & $\mathrm{M}$ & $\mathrm{M}$ & $\mathrm{FL}$ \\
\hline$x_{2}$ & $\mathrm{FH}$ & $\mathrm{FH}$ & $\mathrm{M}$ \\
\hline$x_{3}$ & $\mathrm{M}$ & $\mathrm{FH}$ & $\mathrm{M}$ \\
\hline$x_{4}$ & $\mathrm{H}$ & $\mathrm{M}$ & $\mathrm{FL}$ \\
\hline
\end{tabular}

The specific calculation steps of the proposed decision making method considering with the psychological behavior of the decision makers are given below:

Step 1. The linguistic terms decision matrices $\tilde{S}^{k}=\left(s_{i j}^{k}\right)_{m \times n}$ are transformed into $\tilde{A}^{k}=\left(\tilde{a}_{i j}^{k}\right)_{m \times n}(k=1,2, \ldots, s)$ and given in Table 5 to Table 7 .

Step 2. The evaluation information of the expert group is gathered and expressed with decision matrix $\tilde{A}=\left(\tilde{a}_{i j}\right)_{m \times n}$, which is shown in Table 8 . 
Table 3: Linguistic evaluation values given by expert 2

\begin{tabular}{|c|c|c|c|}
\hline Alternatives & $o_{1}$ & $o_{2}$ & $o_{3}$ \\
\hline$x_{1}$ & $\mathrm{FL}$ & $\mathrm{M}$ & $\mathrm{L}$ \\
\hline$x_{2}$ & $\mathrm{FH}$ & $\mathrm{H}$ & $\mathrm{M}$ \\
\hline$x_{3}$ & $\mathrm{M}$ & $\mathrm{FH}$ & $\mathrm{FL}$ \\
\hline$x_{4}$ & $\mathrm{H}$ & $\mathrm{FH}$ & $\mathrm{FL}$ \\
\hline
\end{tabular}

Table 4: Linguistic evaluation values given by expert 3

\begin{tabular}{|c|c|c|c|}
\hline Alternatives & $o_{1}$ & $o_{2}$ & $o_{3}$ \\
\hline$x_{1}$ & $\mathrm{M}$ & $\mathrm{FH}$ & $\mathrm{FL}$ \\
\hline$x_{2}$ & $\mathrm{M}$ & $\mathrm{FH}$ & $\mathrm{M}$ \\
\hline$x_{3}$ & $\mathrm{FH}$ & $\mathrm{FH}$ & $\mathrm{M}$ \\
\hline$x_{4}$ & $\mathrm{H}$ & $\mathrm{H}$ & $\mathrm{M}$ \\
\hline
\end{tabular}

Table 5: Trapezoidal intuitionistic fuzzy decision matrix given by expert 1

\begin{tabular}{|c|l|l|l|}
\hline Alternatives & \multicolumn{1}{|c|}{$o_{1}$} & \multicolumn{1}{c|}{$o_{2}$} & \multicolumn{1}{c|}{$o_{3}$} \\
\hline$x_{1}$ & $<(0.3,0.4,0.5,0.6)$, & $<(0.3,0.4,0.5,0.6)$, & $<(0.1,0.2,0.3,0.4)$, \\
& $(0.2,0.4,0.5,0.7)>$ & $(0.2,0.4,0.5,0.7)>$ & $(0.0,0.2,0.3,0.5)>$ \\
\hline$x_{2}$ & $<(0.5,0.6,0.7,0.8)$, & $<(0.5,0.6,0.7,0.8)$, & $<(0.3,0.4,0.5,0.6)$, \\
& $(0.4,0.6,0.7,0.9)>$ & $(0.4,0.6,0.7,0.9)>$ & $(0.2,0.4,0.5,0.7)>$ \\
\hline$x_{3}$ & $<(0.3,0.4,0.5,0.6)$, & $<(0.5,0.6,0.7,0.8)$, & $<(0.3,0.4,0.5,0.6)$, \\
& $(0.2,0.4,0.5,0.7)>$ & $(0.4,0.6,0.7,0.9)>$ & $(0.2,0.4,0.5,0.7)>$ \\
\hline$x_{4}$ & $<(0.7,0.8,0.9,1.0)$, & $<(0.3,0.4,0.5,0.6)$, & $<(0.1,0.2,0.3,0.4)$, \\
& $(0.7,0.8,0.9,1.0)>$ & $(0.2,0.4,0.5,0.7)>$ & $(0.0,0.2,0.3,0.5)>$ \\
\hline
\end{tabular}

Table 6: Trapezoidal intuitionistic fuzzy decision matrix given by expert 2

\begin{tabular}{|c|l|l|l|}
\hline Alternatives & \multicolumn{1}{|c|}{$o_{1}$} & \multicolumn{1}{c|}{$o_{2}$} & \multicolumn{1}{c|}{$o_{3}$} \\
\hline$x_{1}$ & $<(0.1,0.2,0.3,0.4)$, & $<(0.3,0.4,0.5,0.6)$, & $<(0.0,0.1,0.2,0.3)$, \\
& $(0.0,0.2,0.3,0.5)>$ & $(0.2,0.4,0.5,0.7)>$ & $(0.0,0.1,0.2,0.3)>$ \\
\hline$x_{2}$ & $<(0.5,0.6,0.7,0.8)$, & $<(0.7,0.8,0.9,1.0)$, & $<(0.3,0.4,0.5,0.6)$, \\
& $(0.4,0.6,0.7,0.9)>$ & $(0.7,0.8,0.9,1.0)>$ & $(0.2,0.4,0.5,0.7)>$ \\
\hline$x_{3}$ & $<(0.3,0.4,0.5,0.6)$, & $<(0.5,0.6,0.7,0.8)$, & $<(0.1,0.2,0.3,0.4)$, \\
& $(0.2,0.4,0.5,0.7)>$ & $(0.4,0.6,0.7,0.9)>$ & $(0.0,0.2,0.3,0.5)>$ \\
\hline$x_{4}$ & $<(0.7,0.8,0.9,1.0)$, & $<(0.5,0.6,0.7,0.8)$, & $<(0.1,0.2,0.3,0.4)$, \\
& $(0.7,0.8,0.9,1.0)>$ & $(0.4,0.6,0.7,0.9)>$ & $(0.0,0.2,0.3,0.5)>$ \\
\hline
\end{tabular}

Step 3. In order to facilitate the comparison with the results of Ye [32], here we also assume that the attribute weights are known with $w_{1}=0.3490, w_{2}=0.3020$ and $w_{3}=0.3490$.

Step 4. For given attitude index value $\lambda=1 / 2$ and $r=0$, the comprehensive evaluation values of the extended TODIM method are calculated as

$$
\xi_{1}=0, \xi_{2}=1.0000, \xi_{3}=0.7421, \xi_{4}=0.4370
$$


Table 7: Trapezoidal intuitionistic fuzzy decision matrix given by expert 3

\begin{tabular}{|c|l|l|l|}
\hline Alternatives & \multicolumn{1}{|c|}{$o_{1}$} & \multicolumn{1}{c|}{$o_{2}$} & $o_{3}$ \\
\hline$x_{1}$ & $<(0.3,0.4,0.5,0.6)$, & $<(0.5,0.6,0.7,0.8)$, & $<(0.1,0.2,0.3,0.4)$, \\
& $(0.2,0.4,0.5,0.7)>$ & $(0.4,0.6,0.7,0.9)>$ & $(0.0,0.2,0.3,0.5)>$ \\
\hline$x_{2}$ & $<(0.3,0.4,0.5,0.6)$, & $<(0.5,0.6,0.7,0.8)$, & $<(0.3,0.4,0.5,0.6)$, \\
& $(0.2,0.4,0.5,0.7)>$ & $(0.4,0.6,0.7,0.9)>$ & $(0.2,0.4,0.5,0.7)>$ \\
\hline$x_{3}$ & $<(0.5,0.6,0.7,0.8)$, & $<(0.5,0.6,0.7,0.8)$, & $<(0.3,0.4,0.5,0.6)$, \\
& $(0.4,0.6,0.7,0.9)>$ & $(0.4,0.6,0.7,0.9)>$ & $(0.2,0.4,0.5,0.7)>$ \\
\hline$x_{4}$ & $<(0.7,0.8,0.9,1.0)$, & $<(0.7,0.8,0.9,1.0)$, & $<(0.3,0.4,0.5,0.6)$, \\
& $(0.7,0.8,0.9,1.0)>$ & $(0.7,0.8,0.9,1.0)>$ & $(0.2,0.4,0.5,0.7)>$ \\
\hline
\end{tabular}

Table 8: Evaluation information of the expert group

\begin{tabular}{|l|l|l|l|}
\hline$x_{i}$ & \multicolumn{1}{|c|}{$o_{1}$} & \multicolumn{1}{c|}{$o_{2}$} & \multicolumn{1}{c|}{$o_{3}$} \\
\hline$x_{1}$ & $<(0.2333,0.3333,0.4333$, & $<(0.3667,0.4667,0.5667$, & $<(0.0667,0.1667,0.2667$, \\
& $0.5333),(0.1333,0.3333$, & $0.6667),(0.2667,0.4667$, & $0.3667),(0.0000,0.1667$, \\
& $0.4333,0.6333)>$ & $0.5667,0.7667)>$ & $0.2667,0.4333)>$ \\
\hline$x_{2}$ & $<(0.4333,0.5333,0.6333$, & $<(0.5667,0.6667,0.7667$, & $<(0.3000,0.4000,0.5000$, \\
& $0.7333),(0.3333,0.5333$, & $0.5667),(0.5000,0.6667$, & $0.6000),(0.2000,0.4000$, \\
& $0.6333,0.8333)>$ & $0.7667,0.9333)>$ & $0.5000,0.7000)>$ \\
\hline$x_{3}$ & $<(0.3667,0.4667,0.5667$, & $<(0.5000,0.6000,0.7000$, & $<(0.2333,0.3333,0.4333$, \\
& $0.6667),(0.2667,0.4667$, & $0.8000),(0.4000,0.6000$, & $0.5333),(0.1333,0.3333$, \\
& $0.5667,0.7667)>$ & $0.7000,0.9000)>$ & $0.4333,0.6333)>$ \\
\hline$x_{4}$ & $<(0.7000,0.8000,0.9000$, & $<(0.5000,0.6000,0.7000$, & $<(0.1667,0.2667,0.3667$, \\
& $1.0000),(0.7000,0.8000$, & $0.8000),(0.4333,0.6000$, & $0.4667),(0.0667,0.2667$, \\
& $0.9000,1.0000)>$ & $0.7000,0.8667)>$ & $0.3667,0.5667)>$ \\
\hline
\end{tabular}

Step 5. Based on the values of $\xi_{i}(i=1,2,3,4)$, the ranking order of the alternatives is obtained as

$$
x_{2}>x_{3}>x_{4}>x_{1},
$$

and $x_{2}$ is the best alternative. This result is in agreement with the one obtained in (Ye [32]).

\section{Conclusion}

Interactive multiple criteria (TODIM) decision method is developed on the basis of the prospect theory, which can describe the psychological behavior of human under uncertain environment, and has been successfully applied to many MADM problems. TODIM method is easier than prospect theory in processing fuzzy numbers, and some authors have already developed it to solve MADM problems in which the attributes values are expressed with crisp numbers, triangular fuzzy numbers, intuitionistic fuzzy numbers, Pythagorean fuzzy and neutrosophic numbers. However, there is no research on the trapezoidal intuitionistic fuzzy environment, and the main work of this paper is to extend TODIM method to solve MADM problems under TIFN environment. First, the article proposes a new class of distance measure of TIFNs, the distance measure can better measure the difference between two TIFNs. Then a new ranking function of TIFNs is introduced, which can take into account the decision-makers' attitude with an attitude index. Finally, the extended TODIM method is put forward to solve the MADM problem in which the attribute evaluation values are expressed with TIFNs. The advantage of this method lies 
in the decision making process which can take into account the decision maker's mentality and the decision maker's perceived value of the gain and loss, so that the decision-making process is more consistent with the objective reality. The proposed distance measure and ranking function can also be used to other MADM methods when the attribute values are expressed with TIFNs.

\section{Funding}

The authors would like to thank the support of the National Natural Science Foundation of China (No.71661012) and Science \& Technology Research Project of Jiangxi Educational Committee (No. GJJ170496 and No. GJJ180829).

\section{Author contributions. Conflict of interest}

The authors contributed equally to this work. The authors declare no conflict of interest.

\section{Bibliography}

[1] Atanassov, K.T. (1986). Intuitionistic Fuzzy Sets, Fuzzy Sets and Systems, 20, 87-96,1986.

[2] Atanassov, K. T.; Gargov, G. (1989). Interval-valued intuitionistic fuzzy sets, Fuzzy Sets and Systems, 31(3), 343-349, 1989.

[3] Bozic, M.; Ducic, N.; Djordjevic, G.; Slavkovic, R. (2017). Optimization of Wheg Robot Running with Simulation of Neuro-Fuzzy Control, International Journal of Simulation Modelling, 16(1), 19-30, 2017.

[4] Dalman, H.; Gazel, N.; Sivri M.(2016). A Fuzzy set-based approach to multi-objective multiitem solid transportation problem under uncertainty, InternationalJournal of Fuzzy Systems, 18(4), 716-729, 2016.

[5] Fan, Z.P.; Zhang, X.; Chen, F.D., Liu, Y. (2013). Extended TODIM method for hybrid multiple attribute decision making problems, Knowlege-Based Systems, 42, 40-48, 2013.

[6] Gomes, L. F. A. M.; Lima, M. M. P. P.(1992). From modeling individual preferences to multicriteria ranking of discrete alternatives: a look at prospect theory and the additive difference model, Foundations of Computing and Decision Sciences, 17, 171-184, 1992.

[7] Gomes, L. F. A. M.; Rangel, L. A. D. (2009). An application of the TODIM method to the multicriteria rental evaluation of residential properties, European Journal of Operational Research, 1193, 204-211, 2009.

[8] Govindan, K.; Jepsen, M. B.(2016). Supplier risk assessment based on trapezoidal intuitionistic fuzzy numbers and ELECTRE TRI-C: a case illustration involving service suppliers, Journal of the Operational Research Society, 67(2), 339-376, 2016.

[9] Grzegorzewski, P. (1998). Metrics and orders in space of fuzzy numbers, Fuzzy sets and Systems, 97(1), 83-94, 1998.

[10] Grzegrorzewski, P. (2003). In ISTANBULL,The Hamming distance between intuitionistic fuzzy sets, Springer, Proc. of the IFSA 2003 World Congress, 35-38, 2003. 
[11] Gu,Y. K.;Zhu, F. L.; Tang, S. Y.(2012). Reliability analysis method based on fuzzy probability importance degree, Journal of Jiangxi University of Science and Technology, 33(5), $51-55,2012$.

[12] Herrera, F.; Viedma, E. H. (2000). Linguistic decision analysis: steps for solving decision problems under linguistic information, Fuzzy Sets and Systems, 115(1), 67-82, 2000.

[13] Kahneman D.; Tversky, A. (1979). Prospect theory: an analysis of decision under risk, Econometrica, 47, 263-292, 1979.

[14] Lakshmana, G. N. V.; Jeevaraj, S.; Dhanasekaran, P. (2016.; A linear ordering on the class of trapezoidal intuitionistic fuzzy numbers, Expert Systems with Applications, 60, 269-279, 2016.

[15] Li, D.-F. (2003). Fuzzy Multiobjective Many-Person Decision Making and Games. National Defense Industry Press, 2003.

[16] Li, X. H.; Chen, X. H. (2015). Multi-criteria group decision making based on trapezoidal intuitionistic fuzzy information, Applied Soft Computing, 30, 454-461, 2015.

[17] Liou, T. S.; Wang, M. J. (1992). Fuzzy weighted average:an improved algorithm, Fuzzy Sets and Systems, 49(3), 307-315, 1992.

[18] Llopis-Albert, C.; Palacios-Marqus, D.; Merigo, J. M. (2016). Decision making under uncertainty in environmental projects using mathematical simulation modeling, Environmental Earth Sciences, 75(19), 1320-1330, 2016.

[19] Lourenzutti, R.; Krohling, R. A. (2014). The Hellinger distance in multicriteria decision making: an illustration to the TOPSIS and TODIM methods, Expert Systems with Applications, 41(9), 4414-4421, 2014.

[20] Mousavi, M.; Yap, H. J.; Musa, S. N.; Dawal, S. Z. M. (2017). A Fuzzy Hybrid GA-PSO Algorithm for Multi-Objective AGV Scheduling in FMS, International Journal of Simulation Modelling, 16(1), 58-71, 2017.

[21] Nehi, H. M.; Maleki, H. R. (2005). In Athens, Intuitionistic fuzzy numbers and it's applications in fuzzy optimization problem, Proceedings of the 9th WSEAS International Conference On Systems, 2005,1-5, 2005.

[22] Pham, V. N.; Long, T. N.; Pedrycz W. (2016). Interval-valued fuzzy set approach to fuzzy co-clustering for data classification, Knowledge-Based Systems, 107, 1-13, 2016.

[23] Qin, Q.; Liang, F.; Li, L.; Wu, G. F. (2017). A TODIM-based multi-criteria group decision making with triangular intuitionistic fuzzy numbers, Applied Soft Computing, 55, 93-10, 2017.

[24] Ren, H. P.; Liu, M. F. (2014). VIKOR method for MADM problem with triangular fuzzy number considering behavior of decision maker, ICIC Express Letters, Part B: Applications, 5(3), 879-884, 2014.

[25] Ren, P.; Xu, Z.; Gou, X.(2016). Pythagorean fuzzy TODIM approach to multi-criteria decision making, Applied Soft Computing, 42, 246-259, 2016 
[26] Sang, X.; Liu, X. (2016). An interval type-2 fuzzy sets-based TODIM method and its application to green supplier selection, Journal of the Operational Research Society, 67(5), 722-734, 2016.

[27] Shu, M. H.; Cheng, C. H.; Chang, J. R.(2006). Using intuitionistic fuzzy sets for fault-tree analysis on printed circuit board assembly, Microelect ronics Reliability, 46(12), 2139-2148, 2006.

[28] Wan, S. P. (2009); Method of attitude index for interval multi-attribute decision-making, Control and Decision, 24(1), 35-38, 2009.

[29] Wan, S. P.; Dong, J. Y. (2015). Power geometric operators of trapezoidal intuitionistic fuzzy numbers and application to multi-attribute group decision making, Applied Soft Computing, $29,153-168,2015$.

[30] Wang, J.; Wang, J. Q.; Zhang, H. Y. (2016). A likelihood-based TODIM approach based on multi-hesitant fuzzy linguistic information for evaluation in logistics outsourcing, Computers \& Industrial Engineering, 99, 287-299, 2016.

[31] Wang, J. Q.; Zhang, Z. (2008). Programming method of multi-criteria decision-making based on intuitionistic fuzzy number with incomplete certain information, Control and Decision, 23(10), 1145-1148, 2008.

[32] Ye, J. (2012). Multicriteria group decision-making method using vector similarity measures for trapezoidal intuitionistic fuzzy number, Group Decision Negotiation, 21, 519-530, 2012.

[33] Zhang, M.; Liu, P.; Shi L. (2016). An extended multiple attribute group decision-making TODIM method based on the neutrosophic numbers, Journal of Intelligent \& Fuzzy Systems, 30(3), 1773-1781, 2016.

[34] Zindani, D.; Maity, S. R. Bhowmik, S.; Chakraborty, S. (2017). A material selection approach using the TODIM (TOmada de Decisao Interativa Multicriterio) method and its analysis, International Journal of Materials Research, 108(5), 345-354, 2017.

[35] Zhu, B.; Xu, Z.S. (2014). A fuzzy linear programming method for group decision making with additive reciprocal fuzzy preference relations, Fuzzy Sets and Systems, 246, 19-33, 2014 . 